\title{
En quête des chefs de dépôt, au temps des compagnies
}

In search of the Depot Heads, at the time of the Companies

\section{Georges Ribeill}

\section{OpenEdition}

\section{Journals}

Édition électronique

URL : https://journals.openedition.org/rhcf/1748

DOI : $10.4000 /$ rhcf. 1748

\section{Éditeur}

Rails \& histoire

\section{Édition imprimée}

Date de publication : 1 décembre 2003

Pagination : 138-168

ISBN : 0996-9403

ISSN : 0996-9403

\section{Référence électronique}

Georges Ribeill, «En quête des chefs de dépôt, au temps des compagnies », Revue d'histoire des chemins de fer [En ligne], 28-29 | 2003, mis en ligne le 17 décembre 2014, consulté le 22 avril 2022. URL : http://journals.openedition.org/rhcf/1748; DOI : https://doi.org/10.4000/rhcf.1748 


\section{Georges RIBEILL}

\section{En quête des chefs de dépôt, au temps des compagnies}

Les dépôts ont toujours constitué un milieu spécifique dans la topographie des réseaux ferroviaires et dans l'univers des métiers du rail. Alors qu'on sait reconstituer les séries de machines propres à chaque réseau ${ }^{1}$, voire les inventaires détaillés et chiffrés des parcs de machines à vapeur gérées par ces établissements ${ }^{2}$, alors que l'on connait bien les étapes successives, caractéristiques, qui ont affecté leur histoire générale des origines à nos jours en raison des nécessaires évolutions techniques, économiques et managériales ${ }^{3}$, on ne dispose toujours pas d'un fichier historique complet de ces établissements voués de manière récurrente à de fortes restructurations, fichier qui signalerait les dates de création, d'éventuelle d'extension... ou de suppression! En particulier, l'archéologie géographique et sociale des dépôts des toutes premières petites compagnies, que viendront bouleverser les fusions ultérieures, reste à faire. Très suggestif de ce paysage ferroviaire originel saupoudré de ces établissements mais aujourd'hui bien oublié est le recensement des dépôts effectué à l'occasion de la grande enquête officielle sur la régularité et la sûreté de l'exploitation, ordonnée en 1853, publiée en 1858... La distinction est bien faite entre les dépôts principaux et les dépôts intermédiaires, « où s’alimentent les machines à leur passage et où, en général, sont placées les machines de réserve et de renfort qui font le service des machines-pilotes », pour reprendre la terminologie des auteurs du Guide du mécanicien ${ }^{4}$.

1- Voir notamment la fameuse série des ouvrages de Lucien-MauriceVilain.

2- Cf. notamment les récents recueils encyclopédiques voués à deux réseaux (fort remarquables mais limités à la vapeur !) : Marcel Chavy; Olivier Constant ; André Rasserie ; José Banaudo, Les Dépôts vapeur du PLM, Breil-sur-Roya, Les Éditions du Cabri, 1997, 303 p. ; Marcel Chavy ; Olivier Constant, Les Dépôts vapeur de l'Ouest, Paris, La Vie du Rail, 2000, 327 p.

3- Cf. la synthèse récente réalisée par Yves Baticle, «Histoire des dépôts de matériel moteur en France (1840-1998) », Revue d'histoire des chemins de fer, $\mathrm{n}^{\circ} 18$ (printemps 1998).

4- Le Châtelier; Flachat; Petiet ; Polonceau, Guide du mécanicien constructeur et conducteur de machines locomotives, Paris, Dupont, Lacroix et Baudry, 1859, p. 418. 
Tableau 1. Répartition géographique des dépôts et leur effectif en 1858.

Source : Enquête sur les moyens d'assurer la régularité et la sûreté de l'exploitation des chemins de fer, Imprimerie impériale, 1858, p. 40.

\begin{tabular}{|c|c|}
\hline Compagnie & Dépôts et réserves \\
\hline \multirow[t]{2}{*}{ Nord } & $\begin{array}{l}\text { Dépôts : Paris, Creil, Saint-Quentin, } \\
\text { Amiens, Boulogne, Douai, Valencienn } \\
\text { Lille, Dunkerque, Calais. }\end{array}$ \\
\hline & $\begin{array}{l}\text { Réserves : Pontoise, Noyon, Breteuil, } \\
\text { Abbeville, Albert, Arras, Hazebrouck. }\end{array}$ \\
\hline \multirow[t]{2}{*}{ Est } & $\begin{array}{l}\text { Dépôts : La Villette, Épernay, Bar-le-DI } \\
\text { Nancy, Strasbourg, Metz. }\end{array}$ \\
\hline & $\begin{array}{l}\text { Réserves : Meaux, Château-Thierry, Vi } \\
\text { Lérouville, Lunéville, Sarrebourg, } \\
\text { Saverne. }\end{array}$ \\
\hline \multirow[t]{2}{*}{ Lyon } & $\begin{array}{l}\text { Dépôts : Paris, Montereau, Tonnerre, } \\
\text { Dijon, Châlons. }\end{array}$ \\
\hline & Réserves : Melun, Sens, La Roche, V€ \\
\hline \multirow[t]{2}{*}{ Orléans } & $\begin{array}{l}\text { Dépôts : Paris, Étampes, Orléans, } \\
\text { Vierzon, Châteauroux, Le Guétin, Moul } \\
\text { Tours, Angers, Nantes, Poitiers, } \\
\text { Angoulême, Bordeaux. }\end{array}$ \\
\hline & $\begin{array}{l}\text { Réserves : Toury, Lamotte, Bourges, } \\
\text { Varennes, Blois, Saumur, Ancenis, Le } \\
\text { Ormes, Ruffec, Chalais, Libourne. }\end{array}$ \\
\hline \multirow[t]{2}{*}{ Méditerranée } & $\begin{array}{l}\text { Dépôts : Marseille, Arles, Avignon, Nîn } \\
\text { Montpellier. }\end{array}$ \\
\hline & $\begin{array}{l}\text { Réserves : Saint-Chamas, Tarascon, } \\
\text { Alais, Cette. }\end{array}$ \\
\hline Ouest & $\begin{array}{l}\text { Dépôts : Vaugirard, Rambouillet, } \\
\text { Chartres, Nogent-le-Rotrou, Le Mans, } \\
\text { Batignolles. }\end{array}$ \\
\hline Rouen-Havr & Dépôts : Batignolles, Sotteville, Le Ha \\
\hline
\end{tabular}

De même, alors que les mécaniciens et chauffeurs constituent deux catégories professionnelles fort bien popularisées, si ce n'est les mieux connues, on connait moins bien les profils professionnels et sociaux des dirigeants de ces établissements positionnés au cœur névralgique de l'exploitation. Alors que l'enquête précitée en livre un 
profil professionnel succinct ${ }^{5}$, leur importance est reconnue, ainsi par Jacqmin $^{6}$, en raison de leurs « attributions très multipliées $»^{7}$ et de leur " grande responsabilité ». À la Compagnie d'Orléans, placés sous les ordres des chefs de traction, les chefs de dépôt commandent les mécaniciens et chauffeurs, les ouvriers chargés de l'entretien des machines et le toutvenant des autres ouvriers moins qualifiés affectés au nettoyage, au chargement du coke, etc. Si l'importance du dépôt le justifie, on adjoint au chef de dépôt, sous ses ordres, un ou plusieurs sous-chefs de dépôt, alors que dans un dépôt d'importance secondaire, ne comprenant par exemple qu'une machine de secours, " on le confie quelquefois à un mécanicien de choix, qu'on relève de temps en temps ${ }^{8}$, ou à un chef de réserve?.

Ces importantes distinctions, fonctionnelles du point de vue technique et hiérarchiques au plan social, sont rarement appréhendées, ou timidement ${ }^{10}$.

5- Cf. la réponse à la question 53 de l'enquête, "Quelles sont les fonctions des chefs de dépôt ? De qui ont-ils des ordres à recevoir ?» : «Les chefs de dépôt surveillent l'entretien des machines; ils sont chargés des petites réparations. Ils sont sous les ordres de l'ingénieur ou de l'entrepreneur de traction et commandent aux mécaniciens. »

6 F. Jacqmin, De l'exploitation des chemins de fer, Paris, Garnier, 1868, tome 1" ${ }^{\text {er }}$ p. 78-79.

7- Attributions ainsi énumérées par F. Jacqmin : «Surveillance de jour et de nuit ; allumage des machines en temps utile et mise en pression ; approvisionnement en eau, combustibles et matières grasses ; surveillance des prises d'eau ; déchargement des wagons de combustible ; lavage des machines, petit entretien, visite des boites ; pose des pièces de rechange ; fourniture des machines demandées par l'exploitation ; trains réguliers, trains supplémentaires, trains en double action ; secours aux trains en détresse ; comptabilité, état du personnel, comptes-matières ; roulement et service des mécaniciens et chauffeurs. »

8- Le Châtelier, Flachat, Petiet, Polonceau, op. cit., p. 412.

9- « Dans les gares moins importantes où il n'existe qu'une réserve de machines, les chefs de dépôt prennent le nom de chefs de réserve » (J.-G. Palaa, Dictionnaire législatif et réglementaire des chemins de fer, Paris, Marchal et Billard, $3^{e}$ édition, 1887, p. 314 ).

10- Révélatrice est la relégation en courte annexe, dans le recueil encyclopédique consacré aux dépôts PLM (M. Chavy et al., op. cit., p. 301), d’une fort intéressante « liste communiquée par Georges Turpin », ventilant en 1915 fonctionnellement les établissements de traction du PLM en six catégories selon le grade de leur responsable: établissements dirigés par un chef de dépôt (au nombre de 30), par un sous-chef de dépót (7), par un chef de réserve (33), par un surveillant chef de réserve (10), par un chef-mécanicien (2), par un surveillant de dépôt enfin (2). 
La variété des charges du chef de dépôt est néanmoins longuement analysée par l'ingénieur centralien Charles Goschler dans son Traité pratique des chemins de fer qui souligne en 1881, au-delà de ses missions techniques, ses impératifs économiques et sociaux ${ }^{11}$ :

Le chef de dépôt est tenu de gérer l'établissement qui lui est confié dans les conditions d'économie la plus scrupuleuse. Il veille, à cet effet, à ce que les machines soient toujours convenablement entretenues, mais sans frais inutiles; que les pièces de rechange ne fassent jamais défaut ; que les approvisionnements de matières de consommation existent toujours en quantités suffisantes, mais sans exagération; que la main-d'œuvre en toute heure réponde strictement aux besoins réels ; enfin, que les agents attachés à son dépôt soient utilement employés, mais en n'exigeant d'eux que le travail normal et en veillant à ce que les conditions hygiéniques soient rigoureusement observées.

Si le chef de dépôt exerce des tâches de commandement vis-àvis de tous les agents du dépôt, roulants, employés, ouvriers et manœuvres, cette mission prend une importance variable en fonction des effectifs et corps d'ouvriers commandés. Le réseau de l'Est sert à Goschler d'exemple concret pour illustrer la gradation d'amplitude importante qui régit le rôle de chef de dépôt au sein d'un même réseau. Ainsi le « dépôt de troisième ordre » de Thionville, succursale de Mohon, qui assure la réserve sur trois directions, occupe en permanence un personnel très réduit : "Un chef de dépôt ${ }^{12}$, un chauffeur, 5 manœuvres et nettoyeurs »; celui « de deuxième ordre » de Givet, à la jonction des lignes de l'Est et du réseau belge, qui possède 20 locomotives, reçoit chaque jour quatre locomotives françaises et 12 belges et assure la réserve pour toutes les lignes qui y aboutissent, occupe un personnel plus varié : « 1 chef de dépôt, 1 sous-chef, 1 comptable, 1 magasinier, 19 mécaniciens et 18 chauffeurs ; 4 chauffeurs de locomobile ; 6 monteurs-ajusteurs, un forgeron, 1 chaudronnier, 1 charron ; 1 lampiste, 2 allumeurs de machines, 10 nettoyeurs et manœuvres. » Le « dépôt de premier ordre » de Nancy, auquel sont attachées 79 locomotives et qui en voit passer 44 autres quotidiennement, se compose de 332 agents

11- Ch. Goschler, Traité pratique de l'entretien et de l'exploitation des chemins de fer, Paris, Baudry, 1881, tome 5, p. 52-54.

12- Aux missions ainsi définies : « visiter et constater l'état des machines en passage ou en stationnement ; prêter aide et assistance aux mécaniciens, s'il y a lieu d'effectuer une petite réparation ; délivrer aux machines de passage les matières qui pourraient leur manquer ; aller au secours des trains en détresse ; enfin surveiller l'état et la marche des machines d'alimentation de la circonscription du dépôt. » 
sous les ordres du chef de dépôt : « 2 sous-chefs, 5 comptables et magasiniers ; 80 mécaniciens et 76 chauffeurs. Ouvriers d'ateliers : 33 monteurs ou ajusteurs, 5 tourneurs, 1 raboteur ; 3 taraudeurs, 2 outilleurs, 6 chaudronniers, 2 forgerons ; 3 charrons, 1 vannier , 18 aides. Hommes de dépôt : 4 lampistes, 12 allumeurs de machines, 18 nettoyeurs, 2 distributeurs, 4 aiguilleurs, 8 chauffeurs de machines fixes ou locomobiles, 5 hommes faisant fonctions de chauffeur, 1 éveilleur, 2 gardiens et 22 manœuvres ou employés divers. »

À noter la fonction de contrôle des roulants dévolue aux chefs mécaniciens. Pour « s'assurer de manière permanente que les mécaniciens accomplissent leurs devoirs avec zèle et intelligence », chefs et souschefs de dépôt sont assistés par des chefs mécaniciens, "suivant les mécaniciens en route, s'assurant qu'ils connaissent parfaitement la conduite et l'alimentation des machines, l'usage et l'observation des signaux, etc. Ces agents supérieurs, chargés également de faire subir aux chauffeurs les examens pour l'admission au grade de mécanicien, font au chef de dépôt, qui les transmet à l'ingénieur de la locomotion, des rapports sur les observations recueillies dans leurs tournées $»^{13}$.

Cette variété des tâches est fort bien soulignée aussi par le publiciste Édouard Charton, dans son guide pour le choix d'une carrière ${ }^{14}$ :

Les chefs de dépôt et, sur une échelle moindre, les chefs de réserve ont des fonctions à la fois administratives et techniques. Ils doivent être constamment prêts à répondre aux besoins de l'exploitation et à fournir les machines qu'elle réclame, complètement équipées, avec leur personnel et leurs approvisionnements. Leurs soins doivent donc se répartir entre le matériel, les agents et les fournitures de toute nature.

Le travail des machines doit être prévu à l'avance, comme celui de leurs conducteurs, et les dispositions doivent être constamment prises de manière à parer, dans le plus bref délai possible, aux accidents qui peuvent se produire. Les approvisionnements nécessaires à la consommation des machines doivent être constamment assurés dans toutes les stations et sur tous les points de ravitaillement. [...] Les chefs de dépôt et de réserve se font suppléer par des sous-chefs, notamment dans les dépôts qui ont un service de nuit.

13- Ibid., p. 54-55.

14- Édouard Charton, Dictionnaire des professions, ou Guide pour le choix d'un état, Paris, Hachette, $3^{e}$ édition, 1880, article "Chemins de fer (personnel des) », p. 122 et sq. 
Alors que l'éventail des appointements au sein de la filière Traction, primes non comprises, est réduit (à la Compagnie de l'Est, en $1880^{15}$ : moyenne mensuelle de $297 \mathrm{~F}$ pour les chefs et sous-chefs de dépôt ; $190 \mathrm{~F}$ pour les mécaniciens, $114 \mathrm{~F}$ pour les chauffeurs), il est plus large s'agissant du montant annuel des primes qui leur reviennent (respectivement de 1200 à $1800 \mathrm{~F}$ pour les chefs et sous-chefs de dépôt; de 300 à $500 \mathrm{~F}$ pour les mécaniciens, de 200 à $300 \mathrm{~F}$ pour les chauffeurs). Pour les primes annuelles revenant aux chefs de dépôt, il s'agit bien de primes de rendement collectif puisque indexées sur le volume des diverses primes gagnées par leurs roulants (économie de combustible, de graisses, prime d'entretien, etc.). L'écart serait plus important encore si l'on tenait compte des avantages en nature comme le logement de fonction ${ }^{16}$.

\section{Des chasses gardées pour certaines écoles d'ingénieurs ?}

Comme le soulignait ce même Édouard Charton ${ }^{17}$, « il n'est pas d'agent supérieur de ce service qui n'ait commencé par être chauffeur et mécanicien, souvent même ouvrier pendant un certain temps ». Et alors que l'enquête de 1853 mettait en avant comme garantie de sûreté de l'exploitation le fait qu'elle fût confiée en grande partie à des agents recrutés parmi des anciens militaires, c'est dans les services du Matériel et de la Traction que ceux-ci étaient les moins nombreux ${ }^{18}$, "les fonctions qu'ont à remplir les mécaniciens [demandant] un apprentissage qu'on ne peut faire dans le régiment ou qui s'oublie, au moins en partie, sous les armes $»^{19}$.

Bien sûr, si l'expérience professionnelle antérieure de chauffeur puis de mécanicien était requise, les dirigeants devaient allier en outre sens du commandement et capacité d'anticipation, comme le suggère le portrait-robot du bon chef de dépôt dressé par Charton : « Tous ces

15- Goschler, op. cit., p. 15.

16- «Les chefs et sous-chefs de dépôt et de réserve ont droit, comme les mécaniciens et les chauffeurs, à des primes d'économie basées sur la bonne gestion des services confiés à leurs soins. Ils sont, en outre, généralement logés, chauffés et éclairés » (Charton, op. cit.)

17- Ibid., p. 122.

18- Selon l'enquête (p. 190), la proportion des agents anciens militaires (soit $2 \times 7$ ans de service) était de $34 \%$ à l'Exploitation, de $29 \%$ à la Voie, de $26 \%$ dans l'administration centrale, et de $18 \%$ seulement au service MT.

19- Enquête..., op. cit., p. IX. 
agents doivent être doués d'un caractère à la fois ferme et bienveillant, comme il convient aux hommes qui ont à diriger un personnel intelligent, à faire souvent appel à son dévouement et à veiller à l'observation de consignes aussi absolues que le sont les consignes militaires. Ils doivent être soigneux, ordonnés, prévoyants, ainsi que l'impose la responsabilité qui leur incombe. »

En fait, si l'accès par la promotion sur le tas fut une nécessité et une option en partie préservée - il était bon de pouvoir offrir aux mécaniciens zélés et dévoués cette perspective d'avancement -, les ingénieurs débutants issus de la jeune École centrale, tout comme les élèves des Écoles des arts et métiers, les gadz'arts, allaient trouver dans l'univers de la Traction un terrain de prédilection professionnelle après avoir rapidement effectué « leurs classes » à la conduite.

«Les élèves de l'École centrale des arts et manufactures, ceux des Écoles d'arts et métiers paraissent particulièrement aptes à remplir les principaux emplois du service du Matériel et de la Traction, car on les trouve dans ce service en plus grand nombre que les anciens élèves de l'École polytechnique. » Cette impression de Charton ${ }^{20}$ est bien confirmée par la réalité : il suffit de feuilleter les premiers annuaires des anciens élèves des ces deux écoles pour y noter, dans la rubrique professionnelle des chemins de fer, leur place prépondérante dans ces services du Matériel (les ateliers de construction et d'entretien du matériel roulant) et de la Traction (les dépôts et leurs "petits entretiens »).

Comme le soulignait en 1859 Perdonnet $^{21}$, l'un des plus anciens professeurs à l'École centrale des arts et manufactures, les centraliens trouvèrent aux débuts des compagnies des situations très élevées dans la hiérarchie de ces deux services. Ainsi Petiet, Polonceau et Mayer occupèrent respectivement au Nord, à l'Orléans et à l'Ouest les plus hautes responsabilités en charge de ces services ${ }^{22}$. Mais il est certain que dans la chasse aux postes de dirigeants, des rivalités d'écoles allaient bientôt opposer les ingénieurs d’État (Ponts et Chaussées, Mines, Génie, Génie

\section{0- Ibid.}

21- Perdonnet, Notions générales de chemins de fer, Paris, Lacroix et Baudry, 1859, p. 141.

22- Bilan favorable aux centraliens, toujours défendu en 1865 par Perdonnet qui est conduit à rectifier un article de Siebecker paru cette année-là dans le Grand Journal : «Quant au service du matériel et de la traction, il est juste de faire connaitre que, sur toutes nos grandes lignes partant de Paris, celle de Lyon-Méditerranée exceptée, il est entièrement dans les mains des élèves de l'École centrale, qui le dirigent en qualité d'ingénieur en chef : M. Petiet au Nord ; M. Villemin, à l'Est ; M. Mayer, à l'Ouest et M. Forquenot à l'Orléans » (Cf. E. Siebecker, Physiologie des chemins de fer, Paris, Hetzel, 1868, p. 69). 
maritime, autres corps militaires) qui avaient pantouflé dans les grandes compagnies aux ingénieurs civils issus d'écoles ou autodidactes. Les pratiques qui en résultèrent de cooptation par le haut s'opposaient à la reconnaissance des talents et des compétences du bas et à de justes promotions, comme le rappelle à mots découverts Perdonnet ${ }^{23}$ :

Le succès des élèves de l'École centrale est d'autant plus remarquable, que, si en France, quelques ingénieurs de l'Etat en ont apprécié le mérite et leur ont accordé une véritable protection, d'autres, en grand nombre, leur sont hostiles et les éloignent autant qu'ils le peuvent du service des compagnies où ils exercent quelque influence; plusieurs, nous regrettons de le dire, hommes de talent cependant et d'un grand mérite, leur refusent leur appui. Il en est de même auxquels ils ont été fort utiles en d'autres temps qui ont aujourd'hui (cela est pénible à dire) perdu entièrement le souvenir de ces anciens services.

La tendance à la marginalisation des autodidactes et des ingénieurs civils titrés dans l'accès aux postes élevés de la hiérarchie des réseaux sera rapide et irréversible, les ingénieurs des Ponts et Chaussées accaparant, après un pantouflage de plus en plus précoce, les postes de direction $^{24}$.

\section{En quête systématique des chefs de dépôt...}

Pour appréhender sociologiquement la population des chefs de dépôt, leurs origines et carrières plus ou moins typiques, encore faut-il en retrouver des listes nominatives, précises et systématiques. Nous ne connaissons qu'une source homogène et globale (traitant de tous les réseaux au même instant) répondant à ces critères, c'est l'Annuaire des chemins de fer Marchal qui, faux « annuaire », ne connaitra qu'un nombre très limité d'éditions entre 1885 et 1934 : de manière détaillée, cet annuaire produit en effet l'organigramme de chaque grande compagnie, jusqu'au bas niveau de ses arrondissements et établissements variés. Une source quasi officielle qui, comme tout annuaire professionnel, peut, à un an près, accuser des retards dans ses mises à jour.

23- Perdonnet, op. cit., 1859, p. 143.

24- Cf. G. Ribeill, La Révolution ferroviaire (1823-1870), Paris, Belin, 1993, p. 317-327; et s'agissant plus précisément des directions des services MT, aussi : "Les ingénieurs en chef du Matériel et de la Traction des grands réseaux ", in B. Escudié ; J. Gréa ; J.-M. Combe (sous la dir. de), Thermodynamique et locomotives à vapeur. L'couvre d'André Chapelon (1892-1978), Éditions du CNRS, 1989, p. 175-195. L'étude des 66 ingénieurs en chef $\mathrm{MT}$ recensés des origines à 1937 révèle le poids croissant des polytechniciens, accédant à ce poste à un âge moyen de plus en plus élevé et mais y restant moins longtemps. 
On a testé l'étude synchronique que permet donc ce document en retenant l'édition de 1912 et en recoupant chaque nom de chef de dépôt indiqué avec les listes d'ingénieurs des annuaires contemporains des Écoles des arts et métiers, de Centrale et de Polytechnique. Le tableau 2 récapitule le résultat de ces recoupements. On a opté pour une présentation par réseau, puis selon l'origine, par promotion pour les chefs de dépôt passés par les écoles citées, alphabétique pour les autres. Sont explicitées certaines ambiguités " géographiques » pouvant relever du décalage des mises à jour entre les divers annuaires ${ }^{25}$.

Tableau 2. Les chefs de dépôt des grands réseaux en 1912.

Sources : Annuaire Marchal, 1912 ; annuaires des anciens élèves des écoles d'ingénieurs, 1912 ; AM Ang : École d'arts et métiers d'Angers ; AM Ch : de Châlons : AM Aix : d'Aix en Provence.

\begin{tabular}{|l|l|l}
\hline Réseau & \multicolumn{1}{|c|}{ Dépôt } & Nom, prénom \\
\hline Est & Longuyon & Jacquet Eugène \\
\hline Est & Verdun & Kern \\
\hline Est & La Villette & Lacombe Louis \\
\hline Est & Châlons & Mielle \\
\hline Est & Amagne & Wauthier \\
\hline Est & Nogent-Vincennes & Courteau Henri \\
\hline Est & Noisy-le-Sec & Berger Eugène \\
\hline Est & Chalindrey & Boeglin Joseph \\
\hline Est & Nancy & Munier Léon \\
\hline Est & Chaumont & Lacombe Élie \\
\hline Est & Bar-le-Duc & Tiphine Charles \\
\hline Est & Épinal & Paris Charles \\
\hline Est & Vesoul & Abram Louis \\
\hline Est & Conflans-Jarny & Marthey Louis \\
\cline { 2 - 3 } & Château-Thierry & \\
\hline Est & Troyes & Groslambert Émile \\
\hline Est & Sézanne & Dieudonné \\
\hline Est & Reims & Deval \\
\hline Est & Château-Thierry & Kieffer \\
\hline
\end{tabular}

25- Ainsi, alors que l'Annuaire Marchal de 1912 indique un Marthey (sans prénom précisé) chef de dépôt à Conflans-Jarny, selon l'Annuaire des gadz'arts de février 1914, il existe un Louis Marthey chef du dépôt de Château-Thierry du même réseau de l'Est. À l'évidence, dans de multiples cas, c'est bien le même individu, saisi à deux moments proches de sa carrière " géographique ». Pour notre traitement statistique, nous considérons qu'il s'agit dans tous les cas du même individu : le risque d'erreur par homonymie et confusion demeure faible. 
(Tableau 2 - suite)

\begin{tabular}{|l|l|l}
\hline Réseau & \multicolumn{1}{|c}{ Dépôt } & Nom, prénom \\
\hline Est & Mohon & Loizillon \\
\hline État & Bressuire & Abel \\
\hline État & Brest & Baroillot \\
\hline État & Orléans & Berthelot \\
\hline État & Châteaubriant & Bigot \\
\hline État & Batignolles-St-Lazare & Boivin \\
\hline État & Argenteuil & Cornette \\
\hline État & Champ-de-Mars & Delhumeau \\
\hline État & Cherbourg & Diringer \\
\hline État & Saintes & Fady \\
\hline État & Aigrefeuille & Gaupuleau \\
\hline État & Thouars & Gérard \\
\hline État & Loudun & Landverlin \\
\hline État & Versailles-Matelots & Langrogne \\
\hline État & Saint-Brieuc & Le Gras \\
\hline État & Sotteville & Leblond \\
\hline État & Lison & Lechien \\
\hline État & Saint-Mariens & Lesné \\
\hline État & Nantes & Letourneur \\
\hline État & La Roche-sur-Yon & Marquet \\
\hline État & Achères & Mauté \\
\hline État & Évreux & Mayot \\
\hline État & Dreux & Mergler \\
\hline ŕat & nannan & nannan \\
\hline
\end{tabular}


(Tableau 2 -suite)

\begin{tabular}{|l|l|l}
\hline Réseau & \multicolumn{1}{|c|}{ Dépôt } & Nom, prénom \\
\hline \multirow{2}{*}{ État } & Segré & Lesueur Gustave \\
\cline { 2 - 3 } & Laval & Weymuller Georges \\
\hline État & Niort & Popineau Lucien \\
\hline État & Batignolles & Seguin Gabriel \\
\hline État & Le Mans & Jeandel Prosper \\
\hline État & Lisieux & Froideval \\
\hline État & Château-du-Loir & Galland \\
\hline État & Mantes & Aubert \\
\hline Midi & Bayonne & Carrière \\
\hline Midi & Tarbes & Chaumerliac \\
\hline Midi & Toulouse & Durmeau \\
\hline Midi & Séverac-le-Château & Huguet Alphonse \\
\hline Midi & Narbonne & Parade Jules \\
\hline Midi & Béziers & Labaig Georges \\
\hline Midi & Bordeaux & Jeanjean Fernand \\
\hline Midi & Castres & Barbieux \\
\hline Nord & Boulogne & Basille \\
\hline Nord & Douai & Bouvier \\
\hline Nord & Cambrai & Cailleret \\
\hline Nord & Arras & Charpentier \\
\hline Nord & Busigny & Denneulin \\
\hline Nord & Valenciennes & Gubucq \\
\hline Nord & Dunkerque & \\
\hline Nord & Laon & \\
\hline
\end{tabular}


(Tableau 2 -suite)

\begin{tabular}{|c|c|c|}
\hline Réseau & Dépôt & Nom, prénom \\
\hline Nord & Creil & Maréchal Victor \\
\hline Nord & Amiens & $\begin{array}{l}\text { Rué Auguste- } \\
\text { Eugène }\end{array}$ \\
\hline \multirow[t]{2}{*}{ Nord } & Aulnoye & \multirow{2}{*}{ Boulanger Ernest } \\
\hline & Vaux-sous-Laon & \\
\hline \multirow[t]{2}{*}{ Nord } & Longueau & \multirow{2}{*}{ Hennebert Jules } \\
\hline & Margny & \\
\hline Nord & La Chapelle & Jacquet Alfred \\
\hline Nord & La Plaine & Gravier Pierre \\
\hline Nord & Fives & Abry Charles \\
\hline Nord & Beaumont & Priez Horace \\
\hline \multirow[t]{2}{*}{ Nord } & Hirson & \multirow{2}{*}{ Delarue Fernand } \\
\hline & Longueau & \\
\hline Nord & Lens & Fourquez André \\
\hline \multirow[t]{2}{*}{ Nord } & Somain & \multirow[t]{2}{*}{ Briet Louis } \\
\hline & Beauvais & \\
\hline \multirow[t]{2}{*}{ Nord } & Hazebrouck & \multirow{2}{*}{$\begin{array}{l}\text { Blanc François } \\
\text { Joseph }\end{array}$} \\
\hline & Somain & \\
\hline PLM & $\begin{array}{l}\text { Le Fayet-Saint- } \\
\text { Gervais }\end{array}$ & Bonjan P. \\
\hline PLM & Grenoble & Bourdier P. \\
\hline PLM & Moulins & Brion L. \\
\hline PLM & Chambéry & Chapel B. \\
\hline PLM & Arenc & Chatillon A. \\
\hline DI $M$ & Mânnn & rnlenn I-R \\
\hline
\end{tabular}


(Tableau 2 -suite)

\begin{tabular}{|c|c|c|}
\hline Réseau & Dépôt & Nom, prénom \\
\hline PLM & Avignon & Prévot $A$. \\
\hline PLM & Chagny & Quoyser \\
\hline PLM & Lons-le-Saulnier & Sales E. \\
\hline PLM & Ambérieu & Vuillard A. \\
\hline \multirow[t]{2}{*}{ PLM } & Dijon-Perrigny & \multirow{2}{*}{ Martin Jules } \\
\hline & Grenoble & \\
\hline PLM & Saint-Étienne & Servoz Jean \\
\hline \multirow[t]{2}{*}{$\overline{\text { PLM }}$} & $\begin{array}{l}\text { Villeneuve-Saint- } \\
\text { Georges }\end{array}$ & \multirow{2}{*}{ Junilhon F. Eugène } \\
\hline & Avignon & \\
\hline \multirow[t]{2}{*}{ PLM } & Nîmes & \multirow{2}{*}{ Pornon Étienne } \\
\hline & Laroche & \\
\hline \multirow[t]{2}{*}{ PLM } & Besançon & \multirow{2}{*}{ Dianoux Jules } \\
\hline & Badan & \\
\hline \multirow[t]{2}{*}{ PLM } & Portes & \multirow{2}{*}{ Salichon Auguste } \\
\hline & Carnoules & \\
\hline PLM & Alais & Canova Élie \\
\hline PLM & Nevers & Méry Mathieu \\
\hline PLM & Annemasse & Billoué Frédéric \\
\hline \multirow[t]{2}{*}{ PLM } & Carnoules & \multirow{2}{*}{ Robin Fernand } \\
\hline & Nevers & \\
\hline \multirow[t]{2}{*}{ PLM } & Badan & \multirow{2}{*}{ Fèvre Louis } \\
\hline & Besançon & \\
\hline PLM & Paris & \\
\hline
\end{tabular}


(Tableau 2 -suite)

\begin{tabular}{|l|l|l}
\hline Réseau & \multicolumn{1}{|c}{ Dépôt } & Nom, prénom \\
\hline PO & Juvisy & Bouyonnet \\
\hline PO & Marmande & Brénac \\
\hline PO & Ussel & Carceller \\
\hline PO & Limoges & Chapoulie \\
\hline PO & Le Mans & Couty \\
\hline PO & Murat & Debrie \\
\hline PO & Nantes & Duroussaud \\
\hline PO & Bergerac & Ecker \\
\hline PO & Châteauroux & Feuillet \\
\hline PO & Périgueux & Gilis \\
\hline PO & Clermont-Ferrand & Gilles \\
\hline PO & Brétigny & Glaume \\
\hline PO & Saint-Nazaire & Grosdemouge \\
\hline PO & Étampes & Guillon \\
\hline PO & Saint-Sulpice & Henry \\
\hline PO & Quimper & Hoguet \\
\hline PO & Blois & Kuypers \\
\hline PO & Orléans & Lautier \\
\hline PO & Montluçon & Leconte \\
\hline PO & Agen & Lefebvre \\
\hline PO & Toulouse & Lefebvre \\
\hline PO & Moulins & Lefèvre \\
\hline PO & Paris & Loiseau \\
\hline PO & Arvant & Masingue \\
\hline & & \\
\hline & & \\
\hline
\end{tabular}


(Tableau 2 -suite)

\begin{tabular}{|l|l|l}
\hline Réseau & Dépôt & Nom, prénom \\
\hline PO & Le Blanc & Roques Édouard \\
\hline PO & Châteaudun & \multirow{2}{*}{ Cochery Georges } \\
& Bort & \\
\hline PO & Poitiers & Hot Charles \\
\hline PO & Capdenac & Moratille Léonard \\
\hline PO & Bordeaux & Lacombe Henri-Adolphe \\
& Brive ? & \\
\hline PO & Bourges & Payssot Joseph \\
\hline PO & Cahors & Pezeu \\
\hline
\end{tabular}

Ce tableau permet de préciser l'importance relative des ingénieurs titrés passés par les dépôts par opposition aux «promus sur le tas $»^{26}$. Parmi les 195 recensés au total, on décompte donc 58 gadz’arts $(29,7 \%$ \%), 3 centraliens et 4 polytechniciens (tous démissionnaires de l'Artillerie), ce qui signifie que ces derniers profils sont moins nombreux et/ou demeurent moins longtemps au poste de chef de dépôt. Le tableau 3 résume le poids relatif des gadz'arts et des «sans titre » promus sur le tas.

Tableau 3. Part des ingénieurs des arts et métiers dans les chefs de dépôts, 1912.

\begin{tabular}{|l|c|c|c|c|c}
\hline & Est & État & Midi & Nord & PLM \\
\hline AM, Aix & 3 & 1 & 4 & 3 & 9 \\
\hline AM, Angers & 1 & 6 & 0 & 1 & 2 \\
\hline AM, Châlons & 6 & 5 & 0 & 8 & 1 \\
\hline \multirow{2}{*}{ AM, total } & $\mathbf{1 0}$ & $\mathbf{1 2}$ & $\mathbf{4}$ & $\mathbf{1 2}$ & $\mathbf{1 2}$ \\
\cline { 2 - 6 } & $\mathbf{( 5 3 \% )}$ & $\mathbf{( 2 9} \%)$ & $\mathbf{( 5 0} \%)$ & $\mathbf{( 3 5} \%)$ & $\mathbf{( 3 4} \%)$ \\
\hline \multirow{2}{*}{ "sans titre » } & 5 & 28 & 4 & 22 & 23 \\
\hline X ou ECP & $(26 \%)$ & $(67 \%)$ & $(50 \%)$ & $(65 \%)$ & $(66 \%)$ \\
\hline Total & $\mathbf{1 9}$ & 2 & 0 & 0 & 0 \\
\hline
\end{tabular}

Sans surprise, on note que le recrutement des gadz'arts favorise sur chaque réseau les ingénieurs issus de l'école la plus proche.

26- Hypothèse bien sûr discutable (il peut y avoir d'autres recrutés sur titres), mais grossièrement vraie... 


\section{La place historique privilégiée des gadz'arts}

En ce qui concerne les gadz'arts, ils furent tôt très nombreux recrutés par les compagnies, occupés aussi bien dans les dépôts de la Traction que dans les ateliers d'entretien et de réparation du Matériel. Cette implantation précoce et variée peut être illustrée à partir des premiers annuaires publiés par la Société des anciens élèves ${ }^{27}$ qui nous livrent, par exemple en 1858, la fonction précise de 55 anciens élèves membres de cette sociéte ${ }^{28}$; soit, classés par compagnie (tabl. 4).

Tableau 4. Les ingénieurs des Arts et Métiers employés dans les compagnies ferroviaires en 1858 .

\begin{tabular}{|l|l|l}
\hline $\begin{array}{c}\text { Prom otion, } \\
\text { école }\end{array}$ & Nom & $\begin{array}{l}\text { Fonction exercée à la } \\
\text { Traction, localité }\end{array}$ \\
\hline 1846, Aix & Rebière & Chef de traction, Paris \\
\hline 1848, Châlons & Curie & $\begin{array}{l}\text { Chef de bureau des } \\
\text { études, Paris }\end{array}$ \\
\hline 1841, Angers & Dumont & Chef de dépôt, Bourges \\
\hline 1824, Angers & Goussard & $\begin{array}{l}\text { Chef de la traction, } \\
\text { Épernay }\end{array}$ \\
\hline 1826, Angers & Rognant & $\begin{array}{l}\text { Chef des ateliers, } \\
\text { Épernay }\end{array}$ \\
\hline 1830, Angers & Robichon & Chef d'atelier, Paris \\
\hline 1831, Angers & Landais & Employé, Epernay \\
\hline 1834, Châlons & Mollard & Chef de traction, Épernay \\
\hline 1840, Châlons & Martin & Ingénieur, Paris \\
\hline 1843, Angers & Dauriat & Chef de dépôt, Bar-le-Duc \\
\hline 1845, Châlons & Dietz & $\begin{array}{l}\text { Chef d'ateliers des } \\
\text { voitures, Metz }\end{array}$ \\
\hline 1845, Châlons & Lambert & $\begin{array}{l}\text { Contre-maître aux ateliers } \\
\text { de carrosserie, Montigny }\end{array}$ \\
\hline 1846, Aix & Imbert & Chef de dépôt, Chaumont \\
\hline 1851, Châlons & Lalevée & $\begin{array}{l}\text { Contrôleur du matériel, } \\
\text { Vrignes-les-Bois }\end{array}$ \\
\hline
\end{tabular}

27- Société des Anciens élèves des Écoles impériales d'Arts et Métiers, Annuaire 1858 publié par le Comité de la Société, Saint-Nicolas près Nancy, 1858 (liste des membres titulaires, p. 21-33).

28- Ce qui fait de cette liste un échantillon peut-être biaisé et non un inventaire complet. 
(Tableau 4. suite)

\begin{tabular}{|c|c|c|}
\hline $\begin{array}{l}\text { Promotion, } \\
\text { école }\end{array}$ & Nom & $\begin{array}{l}\text { Fonction exercée à } \\
\text { Traction, localité }\end{array}$ \\
\hline 1840, Châlons & Gillet-Vital & Chef de service \\
\hline 1837, Châlons & Pètre & Caissier, Paris \\
\hline 1838, Angers & Caillet & $\begin{array}{l}\text { Régisseur des ateliers, } \\
\text { Oullins }\end{array}$ \\
\hline 1842, Angers & Pradel & $\begin{array}{l}\text { Chef de traction, Clerm } \\
\text { Ferrand }\end{array}$ \\
\hline 1848, Châlons & D'Heurs & Dessinateur, Paris \\
\hline 1852, Aix & Didon & Dessinateur, Paris \\
\hline 1839, Châlons & Strohl & Chef des ateliers, Arles \\
\hline 1844, Angers & Longuet & Chef de dépôt, Arles \\
\hline 1840, Châlons & Perret & $\begin{array}{l}\text { Chef de l'atelier des } \\
\text { machines, Bordeaux }\end{array}$ \\
\hline 1841, Angers & Châtelier & $\begin{array}{l}\text { Sous-chef de dépôt, } \\
\text { Toulouse }\end{array}$ \\
\hline 1845, Châlons & Dormoy PJ & $\begin{array}{l}\text { Chef d'ateliers des voitı } \\
\text { Bordeaux }\end{array}$ \\
\hline 1847, Châlons & Schor & $\begin{array}{l}\text { Chef de bureau des étı } \\
\text { Bordeaux }\end{array}$ \\
\hline 1850, Angers & Comairas & Dessinateur, Bordeaux \\
\hline
\end{tabular}


(Tableau 4. suite)

\begin{tabular}{|l|l|l}
\hline $\begin{array}{c}\text { Prom otion, } \\
\text { école }\end{array}$ & Nom & \multicolumn{1}{|c}{$\begin{array}{c}\text { Fonction exercée à la } \\
\text { Traction, localité }\end{array}$} \\
\hline 1852, Angers & Roy Ernest & Employé, Tours \\
\hline 1835, Châlons & Schoedelin & Chef de dépôt, Chartres \\
\hline 1836, Châlons & Caisso & $\begin{array}{l}\text { Sous-chef de traction, } \\
\text { Batignolles }\end{array}$ \\
\hline 1838, Angers & Montouan & Chef de dépôt, Le Mans \\
\hline 1840, Angers & $\begin{array}{l}\text { Cousin- } \\
\text { Lalande }\end{array}$ & $\begin{array}{l}\text { Employé au chemin de fer } \\
\text { Châteaulin, Vannes }\end{array}$ \\
\hline 1842, Châlons & Vidard & Inspecteur du matériel rou \\
\hline 1843, Châlons & Bazaille & $\begin{array}{l}\text { Chef de l'Atelier des } \\
\text { machines, Vaugirard }\end{array}$ \\
\hline 1843, Châlons & Bernier JL & Sous-chef de dépôt, Le N \\
\hline 1845, Chalôns & Cavelan & Chef de dépôt, Le Mans \\
\hline 1833, Angers & Delpech & Ingénieur des ateliers, Par \\
\hline 1842, Châlons & Deligny & Sous-chef d'atelier, Bercy \\
\hline 1850, Angers & Messager & (?), Paris \\
\hline 1840, Châlons & Tourdot & Chef de dépôt, Dijon \\
\hline 1846, Angers & Pottier & Mécanicien, Mâcon \\
\hline 1838, Châlons & Jobert & Employé, Givors \\
\hline 1846, Châlons & Moranges & $\begin{array}{l}\text { Conducteur de travaux, S } \\
\text { Étienne }\end{array}$ \\
\hline 1837, Châlons & Tapet & Chef de section, Bourgoir \\
\hline 1854, Aix & Chrétien & $\begin{array}{l}\text { Chef d'atelier aux forges I } \\
\text { la gare de Saint-Ouen }\end{array}$ \\
\hline
\end{tabular}


En pointant les gadz'arts employés dans les services MT de compagnies françaises ou étrangères, recensés en fonction en 1858 et/ou en 1870, les courtes notices développées dans la liste complète rétrospective des anciens élèves publiée en 1923 permettent d'esquisser un échantillon de leurs trajectoires professionnelles résumées par une fonction et leur employeur. En se limitant aux patronymes commençant par l'une des quatre premières lettres de l'alphabet (de A à D), le tableau 5 révèle quelques caractéristiques importantes de la population des gadz’arts employés dans les chemins de fer.

Tableau 5. Carrières d'ingénieurs des Arts et Métiers dans les services Matériel et Traction (échantillon; avant 1923).

Source : Société des anciens élèves des ENAM, Liste générale décennale alphabétique et par promotions des ancien élèves des ENAM, 1922-1923, Paris, Chaix, 1923.

\begin{tabular}{|c|c|c|}
\hline Promotion/école & Nom, prénom & \\
\hline $\begin{array}{l}\text { s : adhésion à la } \\
\text { SAE }\end{array}$ & (+ décès) & Fonctions exer \\
\hline $\begin{array}{l}1854, \text { Angers } \\
\text { s 1865-1876 }\end{array}$ & Abrieux Étienne & Sous-chef de dépôt, $P_{t}$ \\
\hline 1856, Châlons & $\begin{array}{l}\text { Auclair Antoine } \\
(+1884)\end{array}$ & $\begin{array}{l}\text { Mécanicien } \\
\text { Sous-chef de dépôt, C } \\
\text { Ferrand } \\
\text { Saint-Étienne, puis Mar }\end{array}$ \\
\hline $\begin{array}{l}\text { 1846, Châlons, } \\
\text { s 1863-1892 }\end{array}$ & Baillet Henri & $\begin{array}{l}\text { Chef des ateliers de la } \\
\text { Bordeaux } \\
\text { Idem en } 1890\end{array}$ \\
\hline 1854, Châlons & Barba Paul & Employé au matériel, $\mathrm{P}_{c}$ \\
\hline $\begin{array}{l}1851, \text { Angers } \\
\text { s 1860-1870 }\end{array}$ & Barbut Jean & $\begin{array}{l}\text { en } 1860, \text { sous-chef de } \\
\text { des chemins de fer d'tt } \\
\text { dans différentes comr }\end{array}$ \\
\hline $\begin{array}{l}\text { 1852, Angers } \\
\text { s } 1865\end{array}$ & \begin{tabular}{|l|} 
Barthélémy \\
Charles \\
$(+1886)$ \\
\end{tabular} & $\begin{array}{l}\text { Sous-chef des ateliers } \\
\text { Valladolid } \\
\text { Ing. chef des ateliers d } \\
\text { Norte }\end{array}$ \\
\hline 1850, Châlons & Baur Joseph & $\begin{array}{l}\text { Sous-chef de dépôt, S } \\
\text { En 1900, chef de tracti } \\
\text { à Vesoul }\end{array}$ \\
\hline
\end{tabular}

Insp. : inspecteur; Ing. : ingénieur ; Mec. : mécanicien. 
(Tableau 5-suite)

\begin{tabular}{|c|c|c|}
\hline Promotion/école & Nom, prénom & \multirow[b]{2}{*}{ Fonctions exi } \\
\hline $\begin{array}{l}\text { s : adhésion à la } \\
\text { SAE }\end{array}$ & (+ décès) & \\
\hline $\begin{array}{l}\text { 1843, Châlons } \\
\text { s } 1846\end{array}$ & Bazaille Antoine & $\begin{array}{l}\text { Débute dans une mi } \\
\text { mécan. de précisior } \\
\text { Nord, puis à Meung. } \\
\text { (ateliers Pombla), er } \\
\text { l'Ouest en } 1853, \mathrm{col} \\
\text { contremaître aux atı } \\
\text { Batignolles } \\
\text { Chef de l'Atelier des } \\
\text { en } 1856, \text { Vaugirarc } \\
\text { Insp. MT; retraite er }\end{array}$ \\
\hline \multirow[t]{3}{*}{ 1848, Châlons } & $\begin{array}{l}\text { Belley Louis- } \\
\text { Charles }\end{array}$ & Sous-chef de dépôl \\
\hline & $(+1880)$ & Chef de dépôt \\
\hline & & Insp. du Matériel \\
\hline 1841, Angers & $\begin{array}{l}\text { Bergeron } \\
\text { Jacques/Gustave }\end{array}$ & $\begin{array}{l}\text { Directeur de l'entrek } \\
\text { générale du chemin } \\
\text { Montluçon } \\
\text { Périjusx? }\end{array}$ \\
\hline \multirow{4}{*}{$\begin{array}{l}\text { 1843, Châlons } \\
\text { s } 1846\end{array}$} & Bernier Jean-Louis & Mécanicien \\
\hline & $(+1872)$ & Mécanicien \\
\hline & & Sous-chef de dépôl \\
\hline & & Chef de dépôt, Le N \\
\hline $\begin{array}{l}\text { 1838, Châlons } \\
\text { s 1862-1871 }\end{array}$ & Berthaut Origène & $\begin{array}{l}\text { Mécanicien, Brest } \\
\text { Chef de dépôt, Bres }\end{array}$ \\
\hline
\end{tabular}

Insp. : inspecteur ; Ing. : ingénieur ; Mec. : mécanicien. 
(Tableau 5-suite)

\begin{tabular}{|c|c|c|}
\hline \multirow{2}{*}{$\begin{array}{l}\text { Promotion/école } \\
\text { s : adhésion à la } \\
\text { SAE }\end{array}$} & Nom, prénom & \multirow[b]{2}{*}{ Fonctions exe } \\
\hline & (+ décès) & \\
\hline \multirow[t]{4}{*}{ 1853, Châlons } & \multirow{4}{*}{$\begin{array}{l}\text { Boulongne Louis } \\
(+1894)\end{array}$} & Dessinateur; \\
\hline & & $\begin{array}{l}\text { inspecteur du matéri } \\
\text { Paris }\end{array}$ \\
\hline & & Retraité en 1881 \\
\hline & & $\begin{array}{l}\text { Propr. cultivateur dar } \\
\text { de-Calais }\end{array}$ \\
\hline $\begin{array}{l}\text { 1858, Angers } \\
\text { s 1864-1870 }\end{array}$ & $\begin{array}{l}\text { Bourgeois } \\
\text { Charles }\end{array}$ & Dessinateur, Paris \\
\hline \multirow{4}{*}{$\begin{array}{l}\text { 1847, Châlons } \\
\text { s 1865-1871 }\end{array}$} & \multirow{4}{*}{$\begin{array}{l}\text { Bourgeois Louis } \\
(+1907)\end{array}$} & $?$ \\
\hline & & Chef de dépôt, Paris \\
\hline & & $\begin{array}{l}\text { Installe et dirige en } 1 \\
\text { moulins à farine dans }\end{array}$ \\
\hline & & $\begin{array}{l}\text { Ing. de traction à Nel } \\
\text { retraite en } 1889\end{array}$ \\
\hline \multirow{3}{*}{$\begin{array}{l}\text { 1835, Angers } \\
\text { s 1846-1872 }\end{array}$} & \multirow{3}{*}{$\begin{array}{l}\text { Bourret Marius } \\
(+1887)\end{array}$} & Chef de dépôt, Oran \\
\hline & & Sous-chef de tractio \\
\hline & & Retraité en 1882. \\
\hline $\begin{array}{l}\text { 1834, Angers } \\
\text { s 1846, } 1862\end{array}$ & $\begin{array}{l}\text { Boutard Adolphe } \\
(+1871)\end{array}$ & \begin{tabular}{|l} 
Ingénieur du matériel \\
Villette
\end{tabular} \\
\hline $\begin{array}{l}1855, \text { Angers } \\
\text { s } 1860\end{array}$ & $\begin{array}{l}\text { Brossé Étienne } \\
(+1911)\end{array}$ & $\begin{array}{l}\text { Sous-chef des étude } \\
\text { Paris }\end{array}$ \\
\hline $\begin{array}{l}1848, \text { Angers } \\
\text { s 1861-1870 }\end{array}$ & Brossier Urbain & Sous-chef de dépôt, \\
\hline
\end{tabular}

Insp. : inspecteur ; Ing. : ingénieur ; Mec. : mécanicien. 
(Tableau 5-suite)

\begin{tabular}{|c|c|c|}
\hline Promotion/école & Nom, prénom & \\
\hline $\begin{array}{c}\text { S: adhésion à la } \\
\text { SAE }\end{array}$ & (+ décès) & Fonctions ex \\
\hline $\begin{array}{l}\text { 1838, Angers } \\
\text { s } 1849\end{array}$ & $\begin{array}{l}\text { Caillet Ferdinand } \\
(+1875)\end{array}$ & $\begin{array}{l}\text { Régisseur des atelie } \\
\text { Administrateur de li } \\
\text { Lille, Paris }\end{array}$ \\
\hline 1836, Châlons & $\begin{array}{l}\text { Caisso Marin } \\
(+1896)\end{array}$ & $\begin{array}{l}\text { Installe pour Halette } \\
\text { machines atmosphé } \\
\ddot{\text { Puis chef de dépôt }} \\
\text { Sous-chef de tracti } \\
\text { Batignolles } \\
\text { Ing. des ateliers, Re }\end{array}$ \\
\hline 1852, Aix & $\begin{array}{l}\text { Cambon } \\
\text { Alphonse/Géraud }\end{array}$ & Chef mécanicien, $\mathrm{O}$ \\
\hline 1845, Châlons & $\begin{array}{l}\text { Camme Alphonse } \\
(+1903)\end{array}$ & $\begin{array}{l}\text { Contrôleur principal } \\
\text { de fer de la Haute-lt }\end{array}$ \\
\hline $\begin{array}{l}\text { 1853, Angers } \\
\text { s } 1860\end{array}$ & $\begin{array}{l}\text { Caplen Ernest } \\
(+1899)\end{array}$ & $\begin{array}{l}\text { Contrôleur du matér } \\
\text { traction, Paris } \\
\text { Ing. civil, représenté }\end{array}$ \\
\hline 1857, Châlons & \begin{tabular}{|l|} 
Cassegrain Henri- \\
Pierre $(+1873)$
\end{tabular} & Sous-inspecteur M \\
\hline $\begin{array}{l}\text { 1848, Angers } \\
\text { s } 1851\end{array}$ & $\begin{array}{l}\text { Castellan Ernest } \\
(+1877)\end{array}$ & $\begin{array}{l}\text { Chef des études du } \\
\text { roulant, Paris }\end{array}$ \\
\hline 1858, Angers & $\begin{array}{l}\text { Castera Alexandre } \\
\text { Édouard (+ 1908) }\end{array}$ & $\begin{array}{l}\text { Chef d'entretien du } \\
\text { Bordeaux } \\
\text { Ingr ppal hon. de la }\end{array}$ \\
\hline
\end{tabular}

Insp. : inspecteur ; Ing. : ingénieur ; Mec. : mécanicien. 
(Tableau 5-suite)

\begin{tabular}{|c|c|c|}
\hline Promotion/école & Nom, prénom & \\
\hline $\begin{array}{l}\text { s : adhésion à la } \\
\text { SAE }\end{array}$ & (+ décès) & Fonctions exe \\
\hline $\begin{array}{l}\text { 1849, Aix } \\
\text { s } 1863\end{array}$ & Chaussegros JB & $\begin{array}{l}\text { Entre en } 1852 \text { au } \\
\text { successivement, } \\
\text { monteur, élève-m } \\
\text { chef de dépôt, } \\
\text { inspecteur de la } \\
\text { Traction, Périgueı } \\
\text { Ingénieur en chef } \\
\text { traction, Ingéniel } \\
\text { chef des ateliers }\end{array}$ \\
\hline $\begin{array}{l}\text { 1854, Aix } \\
\text { s } 1857\end{array}$ & Chrétien Jean & $\begin{array}{l}\text { Chef d'atelier aux } \\
\text { de la gare de Sail } \\
\text { Ingénieur civil, Pa } \\
\text { (SICF) }\end{array}$ \\
\hline $\begin{array}{l}\text { 1837, Châlons } \\
\text { s } 1850\end{array}$ & $\begin{array}{l}\text { Claudel Charles } \\
(+1901)\end{array}$ & $\begin{array}{l}\text { Directeur adjoint } \\
\text { ateliers de constr } \\
\text { Vienne }\end{array}$ \\
\hline 1853, Aix & $\begin{array}{l}\text { Clavel Léon } \\
(+1889)\end{array}$ & $\begin{array}{l}\text { Mécanicien, puis } \\
\text { de la traction, Rei } \\
\text { Chef de dépôt, Tr }\end{array}$ \\
\hline $\begin{array}{l}\text { 1855, Châlons } \\
\text { s } 1885\end{array}$ & $\begin{array}{l}\text { Colin Alfred } \\
(+1900)\end{array}$ & $\begin{array}{l}\text { Employé à la Trac } \\
\text { Lagny } \\
\text { puis Marine aux E } \\
\text { Unis }\end{array}$ \\
\hline
\end{tabular}

Insp. : inspecteur ; Ing. : ingénieur ; Mec. : mécanicien. 
(Tableau 5-suite)

\begin{tabular}{|c|c|c|}
\hline Promotion/école & Nom, prénom & \\
\hline $\begin{array}{l}\text { s: adhésion à la } \\
\text { SAE }\end{array}$ & (+ décès) & Fonctions exe \\
\hline $\begin{array}{l}\text { 1849, Aix } \\
\text { s } 1866\end{array}$ & Couderc Charles & $\begin{array}{l}\text { Sous-chef de dépi } \\
\text { Bordeaux }\end{array}$ \\
\hline & $(+1904)$ & $\begin{array}{l}\text { puis propriétaire à } \\
\text { Bordeaux }\end{array}$ \\
\hline $\begin{array}{l}\text { 1853, Châlons } \\
\text { s } 1863\end{array}$ & $\begin{array}{l}\text { Coulle Cyprien } \\
(+1880)\end{array}$ & $\begin{array}{l}\text { Mécanicien, Mulhoı } \\
\text { Chef de dépôt, Épi }\end{array}$ \\
\hline 1860, Châlons & Courtot Pierre & $\begin{array}{l}\text { Mécanicien, Never } \\
\text { En 1873, méc. à Cl } \\
\text { Ferrand, } \\
\text { Chef de dépôt à Cé }\end{array}$ \\
\hline 1831,Châlons & Danne & $\begin{array}{l}\text { Machiniste de rés } \epsilon \\
\text { Brive }\end{array}$ \\
\hline 1843, Angers & Dauriat Mathurin & $\begin{array}{l}\text { Chef de dépôt, Bar } \\
\text { Chef de dépôt, Bar }\end{array}$ \\
\hline 1843, Angers & Dauriat Mathurin & Chef de dépôt, $\mathrm{Bar}$ \\
\hline 1839, Châlons & Decourt Pierre & $\begin{array}{l}\text { Inspecteur de la Tr } \\
\text { Le Mans }\end{array}$ \\
\hline 1848, Châlons & \begin{tabular}{|l} 
Delarbre Charles- \\
Nicolas
\end{tabular} & Dessinateur MT, M \\
\hline 1846, Châlons & $\begin{array}{l}\text { Delbarre Isaîe- } \\
\text { Joseph }\end{array}$ & Chef de dépôt, Ve: \\
\hline 1842, Châlons & Deligny & Sous-chef d'atelier \\
\hline $1833, \mathrm{An}$ & Delpech aîné & Ingé \\
\hline
\end{tabular}

Insp. : inspecteur ; Ing. : ingénieur ; Mec. : mécanicien. 
(Tableau 5-suite)

\begin{tabular}{|l|l|l}
\hline $\begin{array}{l}\text { Prom otion/école } \\
\text { s : adhésion à la } \\
\text { SAE }\end{array}$ & Nom, prénom & (+ décès) \\
\hline 1845, Châlons & Dietz David aîné & $\begin{array}{l}\text { Ing. des ateliers, I } \\
\text { les-Metz }\end{array}$ \\
\hline 1845, Châlons & Dormoy Pierre J & $\begin{array}{l}\text { Chef d'ateliers de! } \\
\text { voitures, Bordeau }\end{array}$ \\
$\begin{array}{l}\text { Constructeur-fonc } \\
\text { bronze et objets o } \\
\text { Bordeaux }\end{array}$ \\
\hline 1863, Châlons & Drouillet & Employé à la Trac \\
\hline 1863, Aix & Dufraine Gustave & Mécanicien, Chau \\
\hline 1841, Angers & Dumont & Chef de dépôt, Bc \\
\hline
\end{tabular}

Insp. : inspecteur ; Ing. : ingénieur ; Mec. : mécanicien.

L'examen de ce tableau fait bien ressortir à la fois la dispersion fonctionnelle et la mobilité géographique des gadz'arts, où l'on entrevoit deux types contrastés de carrière : l'une au sein d'une même compagnie, où les promotions successives permettent parfois de gagner de hautes responsabilités, l'autre plus instable, qui bénéficie des opportunités d'embauche par des réseaux implantés notamment à l'étranger.

En France, le PLM fut un grand recruteur de gadz'arts ${ }^{29}$, notamment issus de l'école d'Aix, le classement de sortie déterminant « statutairement » certains privilèges accordés en début de carrière. En fait, Matériel et Traction se partageaient les jeunes recrues, le Matériel offrant, semble-t-il, des faveurs supplémentaires : les siennes étaient choisies parmi les 40 premiers sortants, des avantages étant réservés aux classés parmi les 10 meilleurs dans le rang de sortie de l'École. Un

29- « En dehors des très grandes villes où existent des écoles de commerce, de comptabilité, en dehors des trois centres d'Aix, Angers et Châlons dont les écoles d'arts et métiers constituent pour les chemins de fer de précieuses pépinières d'agents techniques, il existe peu de ressources", rappelle le directeur du PLM Noblemaire (cf. Hommes et choses des chemins de fer, Paris, Dupont, 1905, p. 77). 
cursus « moyen» s'offrait à ces recrues ${ }^{30}$ : ouvriers dans l'un des ateliers spécialisés de la compagnie (Machines, Voitures ou Wagons), payés à la journée, selon le classement et l'affectation, de $5 \mathrm{~F}$ à $6,50 \mathrm{~F}$, puis au bureau des Études à Paris comme dessinateurs (traitement de $1800 \mathrm{~F}$, élevé à $2100 \mathrm{~F}$ pour les 10 premiers), puis retournant dans l'un des ateliers, comme contrôleurs ou contremaitres. Un cursus parallèle se déroulait à la Traction : stage initial d'ajusteurs dans les dépôts des machines ; puis, une fois remplies certaines conditions, passage à la conduite comme chauffeurs (de 1500 à $1800 \mathrm{~F}$ par an), puis mécaniciens (traitement de $2100 \mathrm{~F}$ à $3000 \mathrm{~F}$, non compris les primes), chefs-mécaniciens (3 $300 \mathrm{~F}$ ) et, pour certains, sous-chefs (de 3600 à $4500 \mathrm{~F}$ ), chefs de dépôt (de 5000 à $6500 \mathrm{~F})$, sous-chefs de traction et même parfois ingénieurs chefs de traction. Alors que l'emploi en début de carrière comme ajusteur dans les entretiens des ateliers était la règle commune de tous les ouvriers recrutés dans les dépôts, y compris pour le tout venant destiné à devenir chauffeurs et mécaniciens, ce qui distinguait bien les gadz'arts c'était tant leur bagage technique acquis sur les bancs de école que le conditionnement réputé à une autorité et à une discipline toutes militaires qu'ils y avaient trouvé, ce qui les prédisposait à devenir plus tard des relais hiérarchiques dévoués et efficaces de leur direction dans ces postes intermédiaires de chefs d'atelier ou de dépôt qui exigeaient un sens certain du commandement $t^{31}$.

Dans la mêlée concurrentielle où s'agitent les mécaniciens candidats à faire une carrière sédentaire dans l'encadrement des dépôts, les gadz'arts avaient certainement quelques atouts personnels et culturels pour se fairevaloir et s'imposer plus facilement que les agents sortis du rang. On a pu ainsi dénoncer le « favoritisme » dont les gadz'arts, « ces fils de parents

30- D'après Paul Blancarnoux, Du choix d'une carrière industrielle, Paris, Dunod, 1904, qui cite, signée du gadz'arts Pierre Blanc (Aix, 1867) qui fut le chef du secrétariat du Matériel et de la Traction au PLM, la Note sur les emplois offerts aux Anciens Élèves des Écoles d'Arts et métiers par le Chemin de fer PLM (Service du Matériel et de la Traction).

31- Selon un article du Bulletin de la Société des anciens élèves (décembre 1879, p. 722), cité par Charles R. Day (Les Écoles d'Arts et Métiers. L'enseignement technique en France, XIX-XX' siècles, Belin, 1991, p. 326), le PLM occupait alors 379 anciens des Arts et Métiers, « la plupart à la division de la Traction, la plus prestigieuse; mais jamais on n’en trouvera plus de $8 \%$ dans les postes les plus élevés d'ingénieur, inspecteur ou chef de division. La plupart devaient se contenter d'emplois de chefs de dépôt, de chefs d'atelier et de contremaitres (44\%), les autres étant mécaniciens ou dessinateurs ». 
aisés ", étaient l'objet de la part des compagnies ${ }^{32}$, ou décoder leur stratégie maritale subordonnée au service de leur avancement ${ }^{33}$ !

L’enquête minutieuse que Blancarnoux a menée en 1904 auprès de camarades affectés comme dirigeants de dépôts (chefs-mécaniciens, sous-chefs et chefs de dépôt) dans diverses compagnies le conduit à souligner la dureté et la pénibilité de ces carrières, voire à afficher un certain scepticisme sur le bilan final ${ }^{34}$ :

Nous ne pouvons conseiller cette orientation qu'à ceux de nos jeunes camarades "robustes et ayant le caractère du métier". Après une antichambre de six mois (environ) dans les ateliers, ils pourront être nommés chauffeurs, ce qui leur permettra, durant deux à quatre trimestres, de toucher, grâce aux économies, une moyenne mensuelle de $200 \mathrm{~F}$.

Les voilà ensuite élèves, puis mécaniciens pour plusieurs années, au cours desquelles ils pourront recevoir de 300 à $500 \mathrm{~F}$ par mois (selon leur ancienneté et surtout la générosité des administrations).

Mais l'emploi est excessivement rude, malgré les périodes assez régulières de courts repos, à cause des incessantes marches de nuit, des services supplémentaires imprévus, des caprices du roulement, de certaines chinoiseries, etc.

Passés chefs-mécaniciens, ils se verront un peu moins bien rétribués, seront astreints à de nombreux "accompagnements", en vue de fréquents rapports (encore l'utilité du style et des racines), sur les essais de charbons, d'huiles et autres.

Enfin, sous-chefs de dépôt vers ou bien avant la trentaine (surtout au Nord, la compagnie actuellement la plus avantageuse), puis chefs de dépôt, ils pourront jouir longuement de "leur jardin" et de diverses douceurs qui leur seront octroyées par MM. les actionnaires, et qu'ils auront cent fois gagnées aux dépens de leur

32- Mesmard, Les Employés des chemins de fer. Critique des abus des grandes compagnies et projet de réformes dans tous les services, Paris, 1892, p. 39.

33- Informateur de l'enquêteur social Zola préparant La Bête humaine, l'ingénieur Lefèvre de la Compagnie de l'Ouest distingue les mécaniciens selon leur double origine possible, identifiant les gadz'arts aux agents « ambitieux, qui peuvent monter en grade, les plus capables ». Alors que " généralement, le mécanicien épouse une ouvrière qu'on ne voit pas, qui lui apporte simplement son manger», «les ambitieux, ceux des écoles, attendent, restent garçon pour épouser une petite bourgeoise, lorsqu'ils sont montés en grade et devenus par exemple chefs de dépôt » (Cf. notes Lefèvre, in Dossier de La Bête humaine, BNF, Département des manuscrits, NAF $\mathbf{n}^{\circ}$ 10272). Bien plus tard, le mécanicien héros du livre de Paul Nizan, Antoine Bloyé (Grasset, 1933), obéira bien à cette stratégie.

34- Blancarnoux, op. cit., p. 198-199. 
santé, au risque de leur vie. C'est suffisant, cela, même pour avoir le droit de prétendre aux postes les plus transcendants. Tous n'y arrivent point, qui pourtant sont très capables et étaient fort robustes.

En terminant, attirons l'attention des favorisés de la nature sur l'avantage sérieux des retraites; des accumulations de primes, d'assurances, de versements divers qui leur permettront, après 55 ans d'âge, d'entrer paisiblement en jouissance de leur vieillesse prématurée, - s'ils ont eu la chance d'y atteindre, ce que nous leur souhaitons de tout cœur...

Conscrit qui m'interroge, je ne suis point partisan des Grandes Administrations ou Compagnies, ah! mais non! Pourtant, si le bruit et l'odeur de ces chemins ferrés te tentent irrésistiblement, réfléchis longtemps, multiplie tes enquêtes auprès des Anciens qui t'ont devancé sur les fournaises roulantes, réfléchis encore ; puis, si tu "en veux" quand même, file au Nord, et demande résolument à "brasser les feux".

Dix ans après, tu pourras peut-être brasser les fleurs de ton jardin. Que ma prédiction s'accomplisse.

\section{Esquisse d'une approche longitudinale des carrières}

Un autre type d'approche, longitudinale cette fois, de la population des chefs de dépôt est rendue possible grâce à l'existence d'un curieux registre conservé au Centre d'archives du personnel de la SNCF à Béziers ${ }^{35}$ et intitulé Élèves d'écoles. Répertoire des agents des grandes écoles entrées au réseau Est au Service MT. Classement par grandes écoles. Il se présente sous la forme de pages à dix colonnes, ainsi définies :

Col. 1 : Nom, prénom.

Col. 2 : Date de naissance.

Col. 3 : Année d'entrée à l'École.

Col. 4 : Observations relatives aux examens de sortie, classement à la sortie.

Col. 5 : Entrée à la Compagnie : date ; qualité, traitement, résidence.

Col. 6 : Ouvrier, chauffeur en régie : date ; qualité, traitement, résidence.

Col. 7 : Chauffeur, mécanicien : date ; qualité, traitement, résidence.

Col. 8 : Sous-chef de dépôt, chef de dépôt, sous-inspecteur, inspecteur : date ; qualité, traitement, résidence.

Col. 9 : Sous-ingénieur, ingénieur : date ; qualité, traitement, résidence. Col. 10 : Observations.

35- Archives SNCF, Centre de Béziers, Registre N² 27 du Réseau de l’Est, Cote 1999/ 005/Livre/070. C'est Madame Lanet, responsable du Centre, qui nous a signalé l'existence et l'intérêt de ce registre. Nous l'en remercions vivement. 
Comme on le voit, s'agissant de ce qu'on appellerait aujourd'hui les attachés embauchés à la Traction par l’Est, le registre est « pré-formaté » selon un déroulement de carrière présupposé standard, plus ou moins abouti jusqu'au rang d'ingénieur. Nous ne ferons ici qu'évoquer l'intérêt de ce document, certes imparfait ${ }^{36}$, mais qui offre l'avantage de présenter un résumé des carrières initiées à la Traction par les diplômés des grandes écoles. Ainsi, à titre d'avant-goût, relevons trois exemples simplifiés de carrière.

- Oblet, né en 1886, sorti de Polytechnique en 1905, est entré à la Compagnie de l'Est en 1908 comme attachéà l'Atelier Épernay ; l'année suivante, il est élève-mécanicien à Noisy, à $175 \mathrm{~F}$ par mois, puis commissionné à $2100 \mathrm{~F}$ par an; en 1910, il est mécanicien au dépôt de La Villette à $2700 \mathrm{~F}$, puis nommé en 1911 sous-chef de dépôt à Troyes, avec le même traitement de $2700 \mathrm{~F}$; il est chef de dépôt principal à Châlons, avec un traitement de 11000 F en 1920, lorsqu'il est affecté à Vesoul comme ingénieur adjoint à $18500 \mathrm{~F}$; un an avant sa retraite en octobre 1939, il était ingénieur en chef attaché à la direction de la Traction de la SNCF.

- Né en 1888, Préclaire, sorti de Centrale en 1908, est affecté comme attaché à l'Atelier Épernay (qui se révèle être le lieu de stage initiatique de la plupart des attachés de l'époque) ; en 1915, il est élèvemécanicien à Mohon, à $3000 \mathrm{~F}$, mécanicien en 1915, puis promu sous-chef de dépôt à Épinal en 1916 à $3100 \mathrm{~F}$; en 1920, il est chef de dépôt de Bar-le-Duc, à $10725 \mathrm{~F}$; mais il démissionne en novembre 1923 pour aller s'occuper de chemins de fer en Chine...

- Né en 1889, Michard, après avoir fait les Arts et Métiers d'Angers puis Sup'Elec, entre en 1909 à l'Est, est nommé chauffeur en 1910, puis mécanicien en 1913 ; en 1916, sous-chef de dépôt à Chaumont, on le retrouve en 1927 chef de dépôt principal à La Villette, avant d'être nommé l'année suivante ingénieur adjoint au Service central à $38000 \mathrm{~F}$, obtenant son grade ultime d'ingénieur en chef adjoint à Paris-Est en 1942 à $73000 \mathrm{~F}$ et terminant sa carrière à la fin de l'année 1944.

Ainsi, d'une catégorie de diplômés à l'autre, un seul coup d'œil sur le taux de remplissage de la colonne $n^{\circ} 9$ est très suggestif des chances inégales d'accès aux grades de sous-ingénieur ou d'ingénieur.

36- On ignore tout des conditions de son initialisation durant les années 1880, comme de celles de son abandon, postérieur à la création de la SNCF, les dernières inscriptions étant faites en $1947 \ldots$ 
Ce registre permet aussi de mieux pondérer le recrutement relatif des diplômés par la Compagnie de l'Est et son évolution durant ses dernières 60 années d'existence. Ainsi, on a dressé le tableau suivant (tabl. 6) dans lequel on a distingué quatre périodes successives de 1881 à 1937.

Tableau 6. Anciens élèves des grandes écoles d'ingénieurs recrutés par la Compagnie de l'Est, 1889-1937.

Source : Archives SNCF, Centre de Béziers, Registre $N^{\circ} 27$ du Réseau de l'Est, Cote 1999/005/Livre/070.

\begin{tabular}{|c|c|c|c|c|}
\hline Années de recrutement & AM & ECP & $\mathbf{X}$ & Total \\
\hline \multirow{2}{*}{$1881-1895$} & 40 & 5 & 2 & \multirow{2}{*}{47} \\
\cline { 2 - 5 } & $85 \%$ & $11 \%$ & $4 \%$ & \\
\hline \multirow{2}{*}{$1896-1914$} & 61 & 10 & 10 & \multirow{2}{*}{81} \\
\cline { 2 - 5 } & $75 \%$ & $12 \%$ & $12 \%$ & \\
\hline \multirow{2}{*}{$1919-1930$} & 54 & 21 & 19 & \multirow{2}{*}{94} \\
\hline \multirow{2}{*}{$1931-1937$} & $57 \%$ & $22 \%$ & $20 \%$ & \\
\hline & 28 & 6 & 6 & \multirow{2}{*}{40} \\
\hline
\end{tabular}

On peut interpréter les chiffres de ce tableau comme révélateurs du standing croissant de la filière de la Traction jusque dans les années 1920 ; de plus en plus concurrencés, les gadz'arts s'en détournent, commençant à démissionner de la compagnie entre 1911 et 1913 (7 démissions), puis encore plus fréquemment dans les années 20 (14 démissions). Si l'on fait appel à nouveau à eux durant les années de crise, mais quand le volume d'embauches a été fortement restreint, alors qu'ils ont démontré depuis longtemps leurs solides capacités éprouvées à faire carrière à la Traction, en particulier à gérer les dépôts, c'est sans doute en raison de leurs prétentions professionnelles et de leurs exigences salariales moins élevées que celles des jeunes centraliens et polytechniciens qui ont du mal alors à trouver un emploi dans l'industrie. Ayant complété pour certains d'entre eux leur formation « mécanique » à Sup'Elec, les progrès de la traction électrique ne peuvent donc en particulier les intimider.

Concluons en ouvrant une voie de recherche : il serait intéressant, à partir de ce registre des attachés Traction de l'Est, d'approfondir l'étude de leurs carrières en fonction des titres initiaux, de comparer leur vitesse relative d'ascension, de mesurer la mobilité géographique et 
le taux des bifurcations vers le Matériel ou des démissions (et d'apprécier le contexte de leurs opportunités), bref d'approfondir la morphologie sociale de ces cadres de la Traction en recourant notamment à leurs dossiers de carrière éventuellement conservés au même centre d'archives SNCF de Béziers. 